The Structure of Superstitious Action - a further analysis of fresh evidence

Martin Bridgstock $^{a}$, Ida Marais ${ }^{b}$, Kylie Sturgess ${ }^{b}$

${ }^{a}$ School of Biomolecular and Physical Sciences, Griffith University, Nathan, Queensland 4111, Australia

${ }^{b}$ Graduate School of Education, The University of Western Australia, Nedlands, Perth, Western Australia 6009, Australia 


\title{
The Structure of Superstitious Action - further analysis of fresh evidence
}

\begin{abstract}
.
Wiseman and Watt's short scales of positive and negative superstitions have attracted attention in the literature. Using a representative survey of the Australian state of Queensland, the six scale items were applied to 1, 243 respondents. Initial investigation using Cronbach's alpha showed that one of the scales did not function properly. A factor analysis suggested that a four-item and a two-item scale best fitted the data. A Rasch analysis of all the items confirmed this, and showed that the conventional five-category response format was not appropriate, and that three categories fit the data better. The main conclusion is that the results do not support the Wiseman-Watt theory of three positive and three negative superstitions. It does not seem advisable to use these scales without substantial reformulation and re-testing.
\end{abstract}

Keywords: Superstition, Rasch model, Positive superstitious beliefs, Negative superstitious beliefs, Scaling, Luck, Item response theory 


\section{Introduction}

In this journal in 2004, Wiseman and Watt noted that superstitions exist in all cultures, proposed a new classification of superstitious behaviour and sought to demonstrate, through statistical analysis, its usefulness. This study evaluates the classification, using new evidence and several analytical techniques.

Wiseman and Watt (2004) distinguished 'positive superstitions,' which seek to improve one’s welfare, from 'negative superstitions,' which seek to guard against bad outcomes. Examples of the former are crossing fingers, touching wood or carrying lucky charms. Examples of the latter are being uneasy about certain numbers, breaking mirrors or walking under ladders. Wiseman and Watt hypothesise that 'Such "positive” superstitions may serve different psychological functions to negative superstitions.' Wiseman and Watt (2004). To demonstrate the usefulness of this classification, Wiseman and Watt formulated two scales of superstitious behaviour and used a large-scale internet survey to show correlations with some standard items, including gender.

The formulation has been widely cited among researchers (eg Andre, 2006; Andre, 2009; Moulding \& Kyrios, 2006; Damish, Stoberock \& Mussweiler, 2010; Williams, Francis \& Robbins, 2007). This is surprising, in view of a number of problems. The two superstition scales have only three items each, and there is no information about their coherence and scaling properties. Further, an internet survey may not be representative of general populations. Finally, although Wiseman and Watt note that superstitions appear in all cultures, their scale items are completely western: it is not 
clear if positive and negative superstitions are expected to appear in other cultures (eg Huang and Teng 2009)

Recently, Dagnall, Parker and Munley (2009) have examined the Wiseman and Watt superstition scales. From their sample of 245 people, recruited by students, they concluded that one of the positive superstition items - concerning lucky charms showed properties which made it a doubtful component of its scale. With this item removed, a two-factor superstition model does appear to fit their data reasonably well. The authors comment that 'Use of Rasch methods for testing seems desirable.' Dagnall et al. (2009). We agree that further testing is necessary, and in this paper we perform several analyses of Wiseman and Watt's superstition scales, using a representative survey population.

\section{Methodology}

Every two years, the Population Laboratory of Central Queensland University conducts a state-wide telephone survey of residents of the Australian state of Queensland. The survey, known as the Queensland Social Survey (QSS), is stratified to ensure its representativeness regarding urban and rural respondents and gender, but is otherwise random. In 2008 a total of 1243 people were surveyed. There was evidence of inadequate sampling in some groups, especially the 18-24 year age bracket, but otherwise the sample appears broadly representative of the state population (Hanley \& Mummery, 2008). Generally, Queensland shares culture and language with countries such as the UK and USA, making this a legitimate area to study the Wiseman-Watt scales. 
To examine Wiseman and Watt's theory, the authors included the six superstition items, with some minor modifications. The items were all rendered into a standard form of "Have you ever . . .” to make frequencies more comparable with each other. In addition, the item “Are you superstitious about the number 13?” was modified to “. . . any number such as 13 or 4 ?” to allow for people from cultures where superstitions attach to numbers other than 13. Each item had five response options: 'Definitely no', 'Probably no’, 'Unsure’, 'Probably yes’ and 'Definitely yes’. The final items appear in Table 1. Virtually all survey respondents answered all the questions.

As a first step, we dichotomised the six items and calculated Cronbach alphas for the two scales. The alpha for the three 'negative' superstitions was .69, which is acceptable for many purposes (Bland \& Altman 1997). The alpha for the three 'positive’ superstitions was .56, which is not acceptable. It seems clear from this simple analysis that Dagnall, Parker and Munley's concerns are justified about the validity of at least one of the scales.

Table 1. Distribution of Answers on the Superstition Scale Items.

\begin{tabular}{|l|c|c|c|c|c|}
\hline Item & $\begin{array}{c}\text { Definitely } \\
\text { yes }\end{array}$ & $\begin{array}{c}\text { Probably } \\
\text { yes }\end{array}$ & $\begin{array}{c}\text { Not } \\
\text { sure }\end{array}$ & $\begin{array}{c}\text { Probably } \\
\text { no }\end{array}$ & $\begin{array}{c}\text { Definitely } \\
\text { no }\end{array}$ \\
\hline $\begin{array}{l}\text { Have you ever avoided walking under a } \\
\text { ladder because it is associated with bad } \\
\text { luck? (Valid responses = 1240) }\end{array}$ & 16.8 & 13.9 & 1.1 & 11.9 & 56.0 \\
\hline $\begin{array}{l}\text { Have you ever felt anxious about breaking } \\
\text { a mirror because it } \\
\text { is thought to cause bad luck? (1239) }\end{array}$ & 8.9 & 9.2 & 1.1 & 17.3 & 63.2 \\
\hline $\begin{array}{l}\text { Have you ever felt superstitious about any } \\
\text { number, such as 13 or 4? (1240) }\end{array}$ & 8.4 & 8.7 & 1.5 & 16.3 & 64.9 \\
\hline $\begin{array}{l}\text { Have you ever said 'fingers crossed' or } \\
\text { actually crossed your fingers? (1243) }\end{array}$ & 43.2 & 28.1 & 1.1 & 8.3 & 19.3 \\
\hline $\begin{array}{l}\text { Have you ever said 'touch wood' or } \\
\text { actually touched or knocked } \\
\text { on wood? (1243) }\end{array}$ & 57.8 & 24.1 & .6 & 3.9 & 13.7 \\
\hline $\begin{array}{l}\text { Have you ever carried a lucky charm or } \\
\text { object? (1242) }\end{array}$ & 14.8 & 8.8 & .9 & 13.5 & 61.9 \\
\hline
\end{tabular}


Data were analysed by factor analysis, consisting of principal components followed by a varimax rotation was performed on the six items, using PASW17. Then, as suggested by Dagnall et al (2009), data were analysed using the modern test theory method of Rasch analysis, which provides a rigorous mathematical approach to examining item response patterns (Bond \& Fox, 2007; Rasch, 1960; Wright \& Masters, 1982).

The Rasch model assumes that the probability of a given respondent affirming an item is a logistic function of the relative distance between the item location and the respondent location on a linear scale or continuum. We use the polytomous Rasch model, also known as the partial credit model (Andrich, 1978; Wright, \& Masters, 1982; Andrich, 2005) and the software RUMM2030 (Andrich, Sheridan \& Luo, 2010). In addition to the item and person location parameters, threshold parameters are estimated for each item. The threshold is the point on the continuum where the probability of a response in either of two adjacent categories is equal.

\section{Results}

The factor analysis showed that two factors accounted for over $66 \%$ of the variance. The factor loadings appear in Table Two. Two slightly correlated factors of superstition are suggested, the first relating to four items, the other relating only to the two others, wood and fingers. As Dagnall et al indicated, charm is anomalous, belonging more to the second factor than to the first. With this change, the four-item superstition scale now has a Cronbach alpha of .69, and the two other items have an alpha of .68. For short scales these are acceptable, though not high. 
Table 2. Factor loadings, after varimax rotation.

\begin{tabular}{|l|c|c|}
\hline Item & Factor I & Factor II \\
\hline Ladder & .797 & .169 \\
\hline Mirror & .843 & .103 \\
\hline Number & .779 & .005 \\
\hline Fingers & .066 & .891 \\
\hline Wood & .125 & .882 \\
\hline Charm & .620 & .066 \\
\hline
\end{tabular}

In sum, a factor analysis does not support the existence of the two superstition scales postulated by Wiseman and Watt. It reveals a different structure, with one four-item factor which may be scalable, and two other correlated items. Possibly the two items wood and fingers are linked because they are very easy to endorse compared to the rest of the items.

For the Rasch analysis, items were rescored, with 'Definitely no', being scored 0 through to 'Definitely yes', being scored 4. The top graph in Figure 1 shows the category probability curves for the item ladder. For data to fit the model each response category should systematically take turns showing the highest probability of endorsement. Figure 1(top) shows that categories 1, 2 and 3 do not have a point where they are the most likely response. In addition, the probability of choosing category 2 is extremely low. This is consistent with previous studies, showing that presenting respondents with a 'Not sure' category as a middle category does not work, and is best presented as a separate response option (Andrich, De Jong \& Sheridan, 1997).

Although the curves for only one item are shown, this pattern was present in all six items. Consequently, all items were rescored by collapsing the 1, 2, and 3 categories 
into one category, creating a total of 3 rather than 5 response categories. Figure 1(bottom) shows that this collapsed structure fitted the model, each category having a point where it is the most likely response.
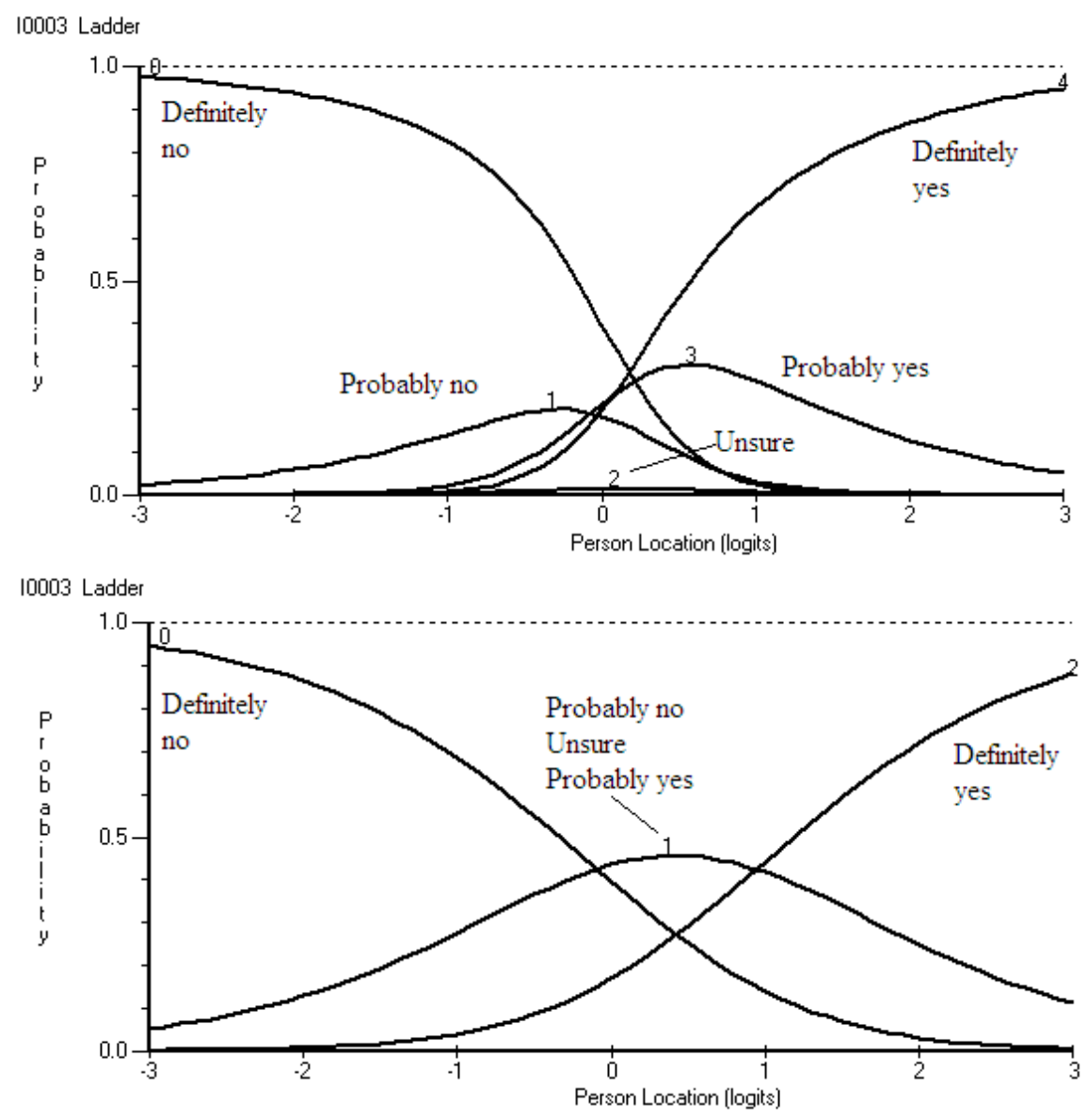

Figure 1 Category probability curves for item 3 before rescoring (top) and after rescoring (bottom)

We also rescored the data using an alternative method. Because the probability of category 2, 'Not sure' was very low, it was rescored as missing data, leaving four response categories for each item. However, the thresholds for all items were still disordered. Items were then further rescored by collapsing the 0 and 1 categories into one 'yes' category, and collapsing the 3 and 4 categories into one 'no' category, creating a total of two response categories. Results of analyses of fit, dimensionality, and DIF based on the second method of rescoring did not differ significantly from the 
first. This structure, however, resulted in low reliability. Hence, the results reported here are based on the rescoring of the original five responses into 3 categories.

Reliability: In RUMM2030 an estimate of the reliability of the scale is available as a person separation index. Under suitable conditions, this is equivalent to Cronbach's alpha (Andrich, 1982). The person separation index was 0.68, which is considered acceptable with so few items.

Person and item locations: Because item, person and threshold estimates are all on the same scale the distributions of persons relative to items or thresholds were examined and indicated that the items aligned well to the persons. Figure 2(left) depicts the distribution of the person locations relative to item locations. A positive value on the continuum indicates persons with a higher level of superstitious belief and items that are difficult to endorse, requiring a high level of superstitious belief. A negative value indicates persons with a lower level of superstitious belief and items that are easy to endorse, not requiring a high level of superstitious belief. The items crossing fingers and touching wood were considerably easier to endorse than the rest of the items. The two most difficult items to endorse were mirror and number, indicating that it takes higher levels of superstitious belief to endorse these items. Figure 2(right) depicts the distribution of the person locations relative to the threshold locations. 

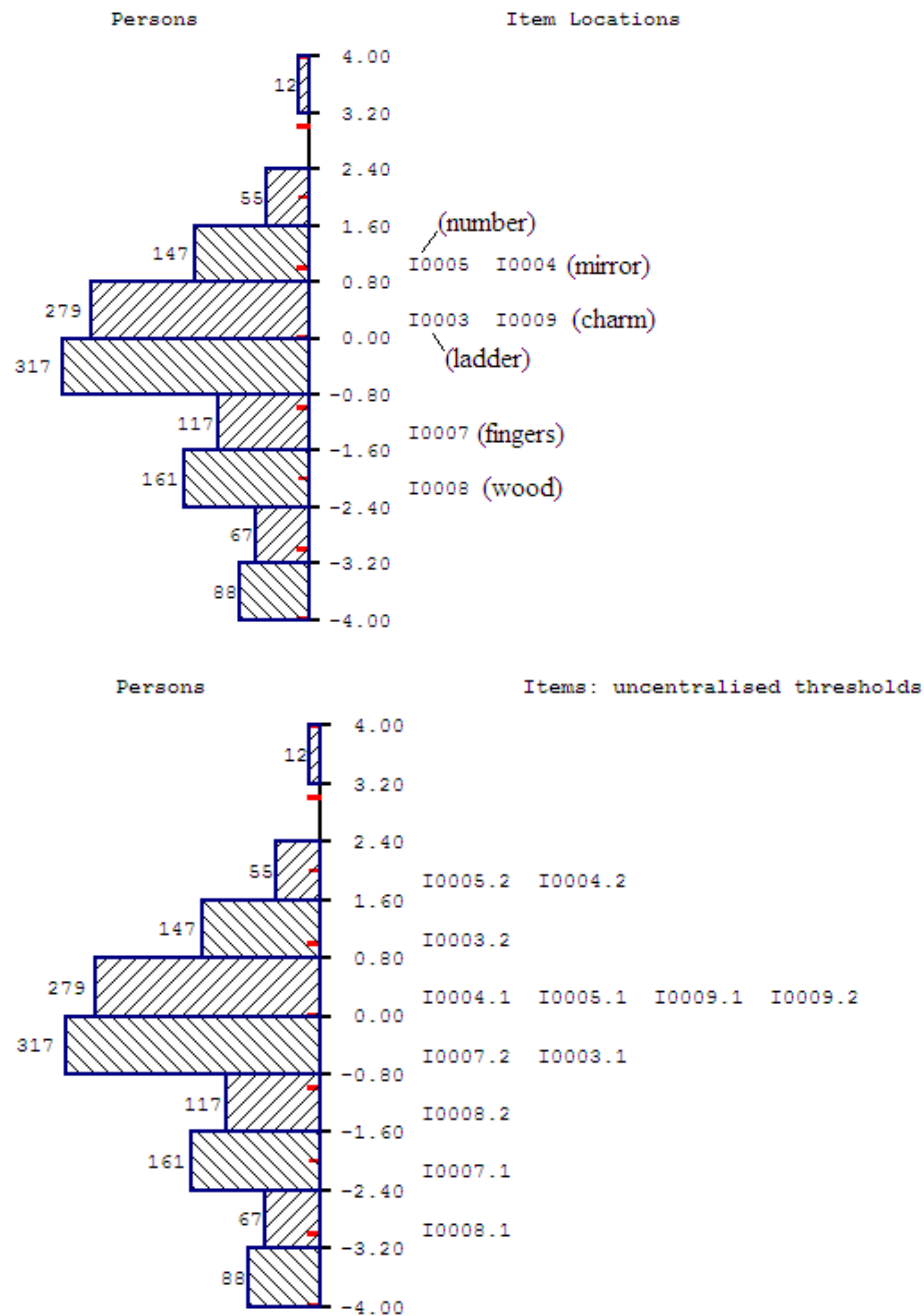

Figure 2 Person item distribution (left) and Person threshold distribution (right)

Differential Item Functioning (DIF) was examined for age and gender groups by conducting an analysis of variance of residuals (ANOVA) for each item comparing scores across each sample group. A residual is the difference between the observed and expected response for a person to an item. The DIF analysis revealed no statistically significant DIF for age or gender.

Item fit \& dimensionality: One of the formal tests of item fit is the chi-square test based on standardised residuals. The mean of the item fit residual values was -0.11 with a SD of 2.66. This deviation was supported by a significant total item-trait 
interaction chi-square statistic of $260.39(\mathrm{df}=24, \mathrm{p}<.01)$. Thus the items do not fit the model. Similarly, the residuals for a person can indicate whether the person fits the model. The mean of the person fit residual values was -0.59 with a SD of 1.41 , indicating no serious misfit of the persons to the model.

Table 2 shows, in addition to item estimates and their standard errors (SE), the individual fit statistics for each item. The individual item fit residuals should range between -2.5 and 2.5 and their associated chi-square statistics should be nonsignificant to indicate fit of a specific item to the model. The items mirror and fingers do not fit the model according to the item fit residual statistic. Mirror overdiscriminates with a fit residual of -3.67. Large negative fit residuals are usually the result of response dependence, which occurs when the response to one item depends on the response to a previous item (Marais \& Andrich, 2008). Fingers underdiscriminates with a fit residual of 3.50. Large positive fit residuals usually indicate multidimensionality, that is, the item measures something different to the rest of the items. Because of the multiple comparisons involved (one test of fit for each item), the alpha level for the chi-square test of fit was adjusted using the Bonferroni correction. This adjusted alpha level was 0.05 / $6=0.0017$ (Bland \& Altman, 1995). No items misfit according to the chi-square test with adjusted alpha level and 4 degrees of freedom (DF).

Table 3. Individual item locations and fit

\begin{tabular}{lrlrrrr} 
Item & Location & SE & FitResidual & ChiSquare & DF & Probability \\
\hline Ladder & 0.42 & 0.05 & -2.29 & 16.16 & 4 & 0.003 \\
Mirror & 1.00 & 0.05 & -3.67 & 17.21 & 4 & 0.002 \\
Number & 0.98 & 0.05 & -0.14 & 6.22 & 4 & 0.183 \\
Fingers & -1.17 & 0.05 & 3.50 & 4.64 & 4 & 0.326 \\
Wood & -1.77 & 0.05 & -0.16 & 8.70 & 4 & 0.069 \\
Charm & 0.55 & 0.05 & 2.13 & 4.03 & 4 & 0.403 \\
\hline
\end{tabular}


Fit of the data to the model was further assessed by examining patterns among the standardized item residuals. First, the correlations between standardized item residuals are shown in Table 3. The only positive correlations were 0.23 between items ladder and mirror and 0.18 between items fingers and wood. These correlations are high relative to the others and indicate violations of the assumption of independence.

Results of a principal component analysis (PCA) of the item residuals are shown in Table 3. After extracting the 'Rasch factor' there should be no further pattern in the data. If a PCA indicates no meaningful pattern the unidimensionality of the scale is supported (Smith, 2002). The results indicated that the loadings directed the items into different sets. Items fingers and wood loaded positively onto the first component and the rest of the items loaded negatively onto this component. The first principal component explained $32.01 \%$ of the total variance among residuals. This suggests multidimensionality, with items fingers and wood tapping into a second factor, after the main factor had been extracted.

Items fingers and wood were both very easy to endorse compared to the rest of the items. In factor analysis items can tend to cluster together because of 'difficulty to endorse' factors even if they measure the same construct as other items (e.g. Nunnally \& Bernstein, 1994 Rasch analysis, however, takes the endorsement pattern of items into account, and the factor structure should not be a result of these patterns.

In addition to the items fingers and wood, items ladder and mirror also violate local independence (as shown in the item residual correlations), but to a lesser extent. In 
addition to the main trait, superstition, these two items also have something in common not shared by the other items.

Table 4. Item residual correlations and results of the PCA

A) Item residual correlations

\begin{tabular}{lcccccc}
\multicolumn{1}{c}{ Item } & 3 & 4 & 5 & 7 & 8 & 9 \\
\hline Ladder & 1 & & & & & \\
Mirror & 0.229 & 1 & & & & \\
Number & -0.108 & 0 & 1 & & & \\
Fingers & -0.302 & -0.361 & -0.341 & 1 & & \\
Wood & -0.239 & -0.329 & -0.325 & 0.181 & 1 & \\
Charm & -0.288 & -0.208 & -0.101 & -0.237 & -0.268 & 1 \\
\hline
\end{tabular}

B) PCA of residuals

\begin{tabular}{lcccccc}
\multicolumn{1}{c}{ Item } & PC1 & PC2 & PC3 & PC4 & PC5 & PC6 \\
\hline Ladder & -0.52 & -0.58 & -0.26 & -0.08 & -0.56 & 0.11 \\
Mirror & -0.66 & -0.35 & -0.14 & 0.14 & 0.63 & 0.10 \\
Number & -0.45 & 0.36 & 0.80 & -0.02 & -0.11 & 0.12 \\
Fingers & 0.74 & -0.19 & 0.06 & 0.63 & -0.05 & 0.13 \\
Wood & 0.68 & -0.30 & 0.10 & -0.62 & 0.17 & 0.12 \\
Charm & -0.03 & 0.83 & -0.54 & -0.07 & -0.03 & 0.14 \\
\hline
\end{tabular}

\section{Discussion and conclusion}

In this paper the Wiseman-Watt scales of 'positive' and 'negative' superstitions, which have achieved considerable acceptance, were tested statistically. Cronbach alphas showed that while the three negative items showed an acceptable level of coherence, the three positive ones did not. A factor analysis revealed that one of the positive items - charm - appeared to belong more with the negative items. However, it is not clear what this four-item scale actually measures, and the remaining two negative items form an extremely short scale.. 
Rasch analysis of the six Wiseman-Watt items also does not support the WisemanWatt theoretical formulation of three positive and three negative superstitions. In particular, items fingers and wood violate local independence (as shown in the item residual correlations) and perhaps tap into a second dimension (as shown by the PCA of the residuals). In addition to the main trait, superstition, these two items have something in common not shared by the other items. The items' ease of endorsement will not lead to this conclusion in a Rasch analysis.

The second main finding of the Rasch analysis is that the five-category response scales used do not appear appropriate. Items essentially functioned as though they had three (or even two), rather than five, response categories. This should be taken into account in future administrations of these items and the response format adapted accordingly. There was no significant Differential Item Functioning (DIF) for either age or gender, though the groups may still differ overall in their levels of superstitious belief.

Most probably, the items wood and finger simply represent precautions in western culture, requiring little belief. The other four items might represent a specifically western superstition scale. Whether counterparts to this exist in other cultures (eg Huang and Teng 2009) is a matter for investigation.

Our conclusion is that the concepts of positive and negative superstitions, and the scales as developed by Wiseman and Watt, are not supported by any of our analyses. 
The most viable scale statistically consists of the three negative items plus the charm item, and its meaning is not clear. In addition there are strong indications that the response categories used are inappropriate. For these reasons, in their present form, we do not regard these scales as being valid measures of superstition. 


\section{References}

Andre, N. (2006). Good fortune, luck, opportunity and their lack: how do agents perceive them? Personality and Individual Differences, 40, 1461-1472

Andre, N. (2009). I am not a lucky person: an examination of the dimensionality of beliefs about chance. Journal of Gambling Studies, 25(4), 473-487

Andrich, D. (1978). A rating formulation for ordered response categories. Psychometrika, 43, 561-573.

Andrich, D. (1982). An index of person separation in latent trait theory, the traditional KR.20 index, and the Guttman scale response pattern. Education Research and Perspectives, 9, 95-104.

Andrich, D. (2005). The Rasch model explained. In S. Alagumalai, D. Curtis \& N. Hungi (Eds.), Applied Rasch Measurement: A Book of Exemplars. (pp. 308-328) Dordrecht: Springer-Kluwer.

Andrich, D., De Jong, J.H.A.L. \& Sheridan, B.E. (1997). Diagnostic Opportunities with the Rasch model for ordered response categories. In J. Rost \& R. Langeheine (Eds.), Applications of Latent Trait and Latent Class Models in the Social Sciences, (pp. 59-70) Munster/New York: Waxmann.

Andrich, D., Sheridan, B. \& Luo, G. (2010). RUMM2030. Perth, Australia: RUMM Laboratory.

Bland, J.M. \& Altman, D.G. (1995). Mutiple significance tests: the Bonferroni method. BMJ 310, 170.

Bland J.M. \& Altman, D. G. (1997) Cronbach’s alpha. BMJ 314, 572. 
Bond, T. G. and Fox, C. M. (2007) Applying the Rasch Model (second edn) Toledo, University of Toledo.

Dagnall, N., Parker A. \& Munley, G. (2009). Assessing Superstitious Belief. Psychological Reports, 104, 447-454.

Damisch, L., Stoberock, B. \& Mussweiler, T. (2010). Keep your fingers crossed! How superstition improves performance. Psychological Science, 20, 1-7.

Hanley, C. \& Mummery, K. (2008). Queensland Social Survey 2008. Final Sampling Report. Rockhampton: Central Queensland University.

Huang, L \& Teng, C. (2009). Development of a Chinese Supersitious Belief Scale. Psychological Reports, 104, 807-819.

Marais, I. \& Andrich, D. A. (2008). Formalising dimension and response violations of local independence in the unidimensional Rasch model. Journal of Applied Measurement, 9, 3, 1-16.

Moulding R. \& Kyrios M. (2006). Anxiety disorders and control related beliefs: the exemplar of Obsessive-Compulsive Disorder (OCD). Clinical Psychology Review 26, 573-583.

Nunnally, J.C. \& Bernstein, I.H. (1994). Psychometric theory. New York: McGrawHill.

Rasch, G. (1960). Probabilistic Models for Some Intelligence and Achievement Tests. Copenhagen: Danish Institute for Educational Research. (Expanded edition 1980). Chicago: MESA Press.

Smith, E. (2002). Detecting and evaluating the impact of multidimensionality using item fit statistics and principal component analysis of residuals. Journal of Applied Measurement, 3, 205-231. 
Williams, E., Francis, L.J. \& Robbins, M. (2007). Personality and paranormal belief: a study among adolescents. Pastoral Psychology, 56, 9-14.

Wiseman, R. \& Watt, C. (2004). Measuring superstitious belief; why lucky charms matter. Personality and Individual Differences, 37, 1533-1541.

Wright, B.D. \& Masters, G.N. (1982). Rating Scale Analysis: Rasch Measurement. Chicago: MESA Press. 


\section{Figure captions}

Figure 1. Category probability curves for item 3 before rescoring (top) and after rescoring (bottom)

Figure 2. Person item distribution (left) and Person threshold distribution (right)

\section{Table captions.}

Table 1. Distribution of Answers on the Superstition Scale Items.

Table 2 Factor Loadings, after Varimax Rotation

Table 3. Individual item locations and fit

Table 4. Item residual correlations and results of the PCA 